\title{
Mental Fatigue after Mild Traumatic Brain Injury in Relation to Cognitive Tests and Brain Imaging Methods
}

\author{
Birgitta Johansson (D) \\ Institute of Neuroscience and Physiology, The Sahlgrenska Academy, University of Gothenburg, \\ 41345 Göteborg, Sweden; birgitta.johansson@neuro.gu.se
}

\begin{abstract}
Most people recover within months after a mild traumatic brain injury (TBI) or concussion, but some will suffer from long-term fatigue with a reduced quality of life and the inability to maintain their employment status or education. For many people, mental fatigue is one of the most distressing and long-lasting symptoms following an mTBI. No efficient treatment options can be offered. The best method for measuring fatigue today is with fatigue self-assessment scales, there being no objective clinical tests available for mental fatigue. The aim here is to provide a narrative review and identify fatigue in relation to cognitive tests and brain imaging methods. Suggestions for future research are presented.
\end{abstract}

Keywords: mild TBI; concussion; mental fatigue; cognition brain imaging

Citation: Johansson, B. Mental Fatigue after Mild Traumatic Brain Injury in Relation to Cognitive Tests and Brain Imaging Methods. Int. J. Environ. Res. Public Health 2021, 18, 5955. https://doi.org/10.3390/ ijerph18115955

Academic Editors: Ben Rattray, Bart Roelands, Kristy Martin and Paul B. Tchounwou

Received: 19 March 2021

Accepted: 29 May 2021

Published: 2 June 2021

Publisher's Note: MDPI stays neutral with regard to jurisdictional claims in published maps and institutional affiliations.

Copyright: (C) 2021 by the author Licensee MDPI, Basel, Switzerland. This article is an open access article distributed under the terms and conditions of the Creative Commons Attribution (CC BY) license (https:// creativecommons.org/licenses/by/ $4.0 /)$.

\section{Introduction}

Traumatic brain injury (TBI) is a common health problem and can lead to temporary or permanent disabilities of cognitive or physical functioning [1-3], and approximately 70-90\% of injuries are mild TBI (mTBI) [4]. Most people recover after an mTBI within one to three months, but those who suffer long-term symptoms experience a reduced quality of life with deterioration of general health, cognitive impairments, higher risk of depression, social isolation, behavioral problems, and an inability to maintain employment status or continue with education [5-8]. Thus, "mild" injury may be anything but mild [8].

After a traffic-related mTBI, 23\% did not recover within a year [9]. An unfavorable outcome was reported for $30 \%$ of all mTBI patients [10]. A review reported that approximately half of the individuals with a single mTBI demonstrated long-term cognitive impairment [11]. A worse outcome can develop after recurrence of mTBI [12]. The assessment and definition of symptoms after an mTBI varies between studies, and symptoms are also common in the population irrespective of whether it is a case of an mTBI. The interaction between pre- and post-biological and psychological factors results in a complex model $[13,14]$, and there is limited evidence for variables as predictors of fatigue [14]. However, in many studies, mental fatigue is not recognized as a separate factor, despite being one of the most distressing and long-lasting symptoms following mTBI $[15,16]$, having an impact on well-being and quality of life [2]. Further research is warranted to better understand the associations between fatigue and pre- and post-morbid variables and for the development of adapted treatment options.

Pathological mental fatigue (not to be confused with non-pathological mental fatigue) is a reduced capacity to continue a mental activity repeatedly. It is a lack of mental energy and a reduced ability to restore the energy in response to performed activity. The mental energy varies depending on the activities performed, but it never returns to normal and is not restored after sleep or an extended rest [17]. Mental fatigue restricts the ability to perform daily activities with work, studies, and social activities, and there is a limited endurance over the course of the day and for weeks and months. The mental fatigue is unlike that normally experienced; rather, it persists as a profound exhaustion over months 
or years, or it is sometimes lifelong. People appear normal, but the fatigue they describe goes far beyond and far deeper than a person without brain fatigue could imagine.

The purpose here is to provide a narrative review of the role of mental fatigue in relation to cognitive tests and brain imaging methods after TBI. Both terms, concussion and mTBI, are used.

\subsection{TBI and Prevalence of Long-Term Fatigue}

The precise estimation of fatigue after mTBI depends on the assessments used. Nonetheless, fatigue is often reported as having a bearing on everyday life. One third of patients who had suffered an mTBI reported fatigue at 6 months as well as a decrease in physical and social activities [18]. A consistently high frequency of fatigue after TBI was found during the first six months after the TBI [19], and 40\% suffered from headache and fatigue one year after mTBI [20]. After 5 years, $73 \%$ were still suffering from fatigue after a TBI, affecting their everyday lives [3]. After ten years, the fatigue was still present, irrespective of injury severity [21]. Improvement was reported during the first year, after which time it was limited [22].

\subsection{Origin of Fatigue after TBI}

The origin of fatigue is not fully understood and is often suggested to be related to multifactorial factors including biological, psychological, and social aspects. Fatigue is thus a concept with a subjective feeling of exhaustion possibly having a biological as well as a psychological origin and can be difficult to manage depending on the adapted strategies used and social factors involved. However, fatigue in neurological disorders is suggested to be related to circuits that connect the basal ganglia, amygdala, thalamus, and frontal cortex [23]. This is supported by brain imaging studies with TBI participants [24-29]. The integration of these areas is important for appropriate behavior and cognitive functioning [30] and also for executive function such as motivation, learning, planning, goal-directed behavior, and emotion regulation [31]. Genetic factors also interact with TBI, and carriers with the APOE epsilon4 allele reported fatigue twice as often after compared with before an mTBI, while no increase in fatigue was found in a non-injured control group [32]. A long-term follow up 1-5 years after a TBI showed a carrier with the APOE epsilon4 allele reporting a lower rating on the Glasgow Coma Scale-Extended (GOSE) [33]. In the study by Ponsford et al., fatigue was not measured, but reduced social and leisure activities from GOSE were related to fatigue after TBI and mTBI [34], and fatigue may have been present among the TBI participants [33].

TBI has a heterogeneous pathology. External mechanical or rotational forces result in focal injuries due to contusion, laceration, intra- and/or extra cerebral haemorrhage, and diffuse brain damage. Metabolic and structural lesions that disrupt the pathways interconnecting the basal ganglia, thalamus, limbic system, and higher cortical centre are suggested to be involved in the pathophysiological process of fatigue in neurological disorders [23]. In multiple sclerosis, fatigue is hypothesized to be related to impaired dopamine communication between the striatum and prefrontal cortex [35].

The relation between mental fatigue and a secondary injury that gives rise to a neurochemical cascade that can induce inflammation, biochemical imbalance, oxidative stress, and extended reduction in glucose metabolism [36] is not well understood. However, a theoretical model for the development of mental fatigue in relation to neuroinflammation and disturbed neurochemical balance is presented [37]. Brain injury in the acute phase causes a neuroinflammation, with microglia and astrocytes forming inflammatory substances, protecting the nerve cells from damage. The cytokines (e.g., Tumor Necrosis Factor-alpha and Interleukin 1-beta) initially attenuate the signaling between the brain cells, which is important in the acute phase when energy is needed to repair the nervous system. If such substances persist after the injury, they may instead lead to reduced efficiency in nerve cell traffic, resulting in impaired cognitive function and perception of mental fatigue [37,38]. 


\subsection{Fatigue Self-Assessment Scales}

The best method measuring fatigue today is self-assessment scales. Several scales exist, although there is no consensus regarding the assessment scales. Fatigue can be divided into state- or trait-fatigue. State-fatigue or fatigability is measured as a reduced performance or a worse perception of fatigue in relation to a specific activity performed. Visual analog scales (VAS) are commonly used for fatigability, but also cognitive tests or brain activation are used. Trait-fatigue is measured with assessment scales as the subjective perception of fatigue over a pre-defined period of time (weeks and months) [39]. In relation to the mental fatigue discussed here, both state- and trait-fatigue are valid. The mTBI individual suffering from long-term or chronic mental fatigue will have a long-lasting fatigue and will not be fully recovered, and the mental fatigue will vary over the course of the day and from one day or week to the next. When suffering from long-lasting mental fatigue, there is also a distinct fatigability in relation to performed mental activities, and it takes a long time to recover from the extra load on the brain. Physical activity has not been extensively investigated in terms of mental fatigue and mTBI, but patients commonly report a worsening of their mental fatigue following physical activity.

\subsection{Emotional Distress and Fatigue}

Emotional and psychological problems before the brain injury as well as stress add to the trauma, and personal coping abilities interact with psychological, biological, and genetic factors $[13,40]$. These need to be taken into account for a multifactorial interactive model for mental fatigue during clinical assessment. Clearly, factors such as depression or unemployment before the injury will persist during recovery. Long-lasting fatigue after an mTBI will affect everyday life, such as not managing work and social activities. This can result in an increase in psychological distress if adequate rehabilitation or helpful personal coping strategies are not in place. People suffering from depression and anxiety prior to a skull trauma more frequently experience long-term complications and psychiatric disorders after a brain injury [41-43]. With regard to executive function, self-report of executive function increased after mild to moderate TBI and was found to be a predictor for depressive symptoms, although this was not related to objective tests for executive function [44]. Fatigue was not measured in the study. Many authors have reported depression to be common after a brain injury, but they have seldom included fatigue in their analyses.

According to Cantor et al. [16], overlapping symptoms between fatigue and depression can give misleading results if the core symptoms are not carefully evaluated. When considering fatigue after a TBI, it is suggested to be related to the brain injury itself [2] rather than as a consequence of anxiety and depression [45], even though emotional distress can aggravate the symptoms of fatigue. Fatigue was also found to uniquely contribute to disability after TBI, measured with the Mayo-Portland Adaptability Inventory, after controlling for injury severity, executive functions, and depression status [46]. Fatigue decreased during the first year after mTBI and depression, insomnia, cognitive difficulties, and work status were related to the fatigue [47]. Adjustment for the overlapping items in the Mental Fatigue Scale (MFS) and Comprehensive Psychopathological Rating Scale (CPRS-depression and anxiety) removed the differences in depression and anxiety between participants with an acquired brain injury (mTBI, stroke) and healthy controls, but not for the mental fatigue [48]. It is important to acknowledge the increased risk for depression and suicide after a TBI [49] and also to understand the significance of this within the context of fatigue. Mental fatigue cannot be explained within the context of emotional distress alone. However, treatments for depression and anxiety are essential, and these can reduce mental fatigue and improve well-being [50,51].

\subsection{Cognitive Function in Relation to Fatigue}

From the book by Lezak et al., p 177 [52], the effort to accomplish ordinary activities after a brain injury is exemplified. 
"Activities that are normally automatic but become effortful after the injury include many that are performed frequently throughout a normal day, such as concentrating, warding off distractions, reading for meaning, doing mental calculations, monitoring ongoing performance, planning the day's activities, attending to conversations at once, or conversing with background noise, etc. It is by little wonder that by late afternoon, if not by noon, many of these patients are exhausted".

Despite the awareness of these problems, it has been difficult to detect such cognitive complaints after mTBI using standard neuropsychological tests, with the result that cognitive difficulties can go undetected [53-55]. In the clinic, many patients who suffer from long-term difficulties after an mTBI may perform within a normal range on cognitive tests, although they may still report mental fatigue, including distractibility and difficulties with reading as well as executive function. Executive function is a broad over-reaching term for cognitive and emotional functions and is suggested to have one of the greatest effects on functional outcomes after a TBI [56], irrespective of TBI severity, having a negative impact on daily living and the ability to go back to work and studies [31]. Executive function is important for the ability to respond adaptively to novel situations and manage emotional and social skills, including verbal reasoning, problem solving, attention control, sustained attention, planning, judgment, decision-making, motivation, impulsivity, resistance to interference, utilization of feedback, multi-tasking, and cognitive flexibility [52,54,55]. From a large-scale study including mild to severe TBI subjects, information processing speed was identified as the domain most strongly associated with daily functioning [57]. Neuropsychological tests have been developed to identify focal and diffuse brain damage rather than to predict how a patient would function in his or her environment [58].

The results reported here include both $\mathrm{mTBI}$ and TBI patients, the subject of few studies (Table 1). Fatigue in a group with mild to severe TBI was related to the speed subscales included in the Cambridge Neuropsychological Test Automated Battery (CANTAB) [59]. A worsening of performance after severe TBI on a dual-task with load on working memory and/or executive control was related to increased mental effort. Mental effort was not related to task difficulty or to performance, and it was suggested that fatigue interferes with performance even in simple and automatic tasks [60]. Patients with severe TBI who have a high baseline fatigue performed less well on the selective attention test with a longer reaction time and more omissions compared to controls. Significant correlations were found in the TBI group between attention performance, mental effort, and subjective fatigue. Depression did not correlate with fatigue [61]. Reduced processing speed (digit symbol-coding, reading speed, trail making test) correlated with mTBI and TBI participants all suffering from mental fatigue. Those participants with mTBI working full-time rated their mental fatigue on a similar high level as participants with mTBI and TBI on sick leave. Unlike in the case of those on sick leave, objective cognitive tests did not differ between controls and those with mTBI who were also working full-time. This was suggested to be related to the mental demands during the day. Those suffering from mental fatigue after an mTBI but who are also working full-time might need to devote more time and effort to mental work [34]. Slower performance on a complex selective attention task was associated with fatigue for mild to severe TBI participants in comparison with controls. It was suggested that tasks with a higher order of attentional demand in everyday life are associated with subjective fatigue, this being irrespective of the effects of mood [62]. In contrast, a group including TBI participants reported increased fatigue after the test session having a slower reaction time compared to controls, although the reaction time remained constant for both groups during the session [25]. A vigilance test with a total duration of approximately 45 min was performed by participants with mild to severe TBI. The TBI group was slower and remained at a similar slower speed throughout the duration of the task. Higher state-fatigue was associated with more omissions for TBI participants. A subgroup of these participants showed a decline in performance, which the authors suggested was associated with the greater psychophysiological cost of striving to maintain a stable performance over time, and this cost was associated with a reported increase 
in fatigue [63]. Reaction time was longer and increased during a 20-min vigilance task for mTBI group, while the reaction time remained stable for the controls [26]. Decreased performance (fatigability) was reported for an mTBI group who described more fatigue than controls in more demanding cognitive tests with simultaneous activation of several cognitive domains (Digit Symbol Substitution Test-DSST) and executive functions (Color Word Test). However, fatigability was not found to be present for less demanding tests involving simple automatic attention [64]. Using the Barrow Neurological Institute (BNI) Fatigue Scale and BNI Screen for Higher Cerebral Functions, the TBI group reported significantly higher levels of fatigue compared to controls. Fatigue was unrelated to injury severity, number of days since injury, cognitive impairment, and gender. The authors suggested regular rest during the day for inpatients in rehabilitation units [65]. During a subacute period, no differences between mTBI and controls in simple reaction time tasks was found, but with increased task complexity, the mTBI group performed more slowly than controls. However, no relation between cognition and fatigue was found [66]. Fatigue and relations to saccadic eye movements and attention in mTBI patients and patients with minor orthopedic injuries early after an mTBI was investigated. The mTBI group reported higher self-rated change in fatigue compared with levels prior to the mTBI and showed more fatigability/state-fatigue measured with cognitive tests. Their state-fatigue correlated with prosaccade latency and cognitive fatigability. In contrast, mTBI trait-fatigue correlated with anxiety and antisaccade latency and variability [67].

Table 1. Research relating fatigue in adults after TBI to cognitive function. Summary of study characteristics, including details on study sample, methods, and results.

\begin{tabular}{|c|c|c|c|c|c|c|}
\hline Reference & $\begin{array}{l}\text { Number of Participants } \\
\text { and } \\
\text { Injury Severity }\end{array}$ & Time Since Injury & $\begin{array}{l}\text { Age } \\
\text { Year Mean } \\
\text { (sd) }\end{array}$ & $\begin{array}{c}\text { Sex } \\
\text { (Males/ } \\
\text { Females) }\end{array}$ & $\begin{array}{l}\text { Cognitive Test } \\
\text { Fatigue Measure }\end{array}$ & Results \\
\hline $\begin{array}{l}\text { Anderson \& } \\
\text { Cockle, } 2021 \text { [66] }\end{array}$ & $\begin{array}{l}84 \mathrm{mTBI} \\
47 \mathrm{HC}\end{array}$ & 60 (11) days & $\begin{array}{l}\operatorname{mTBI} 37(14) \\
\text { HC } 34(10)\end{array}$ & $\begin{array}{l}\text { mTBI } 61 / 23 \\
\text { HC } 18 / 29\end{array}$ & $\begin{array}{l}\text { Symbol Digit } \\
\text { Modality Test, } \\
\text { N-back test and a } \\
\text { Increasing Distractors } \\
\text { Paradigm assessing } \\
\text { reaction time under } \\
\text { conditions of } \\
\text { increasing cognitive } \\
\text { load. } \\
\text { MFI }\end{array}$ & $\begin{array}{l}\text { No differences between } \\
\text { mTBI and controls on } \\
\text { simple tasks, but with } \\
\text { increased task complexity, } \\
\text { the mTBI group performed } \\
\text { more slowly than controls } \\
(p<0.05) \text {. The difference in } \\
\text { cognitive performance was } \\
\text { unrelated to fatigue. }\end{array}$ \\
\hline $\begin{array}{l}\text { Ashman et al., } \\
2008 \text { [59] }\end{array}$ & $\begin{array}{l}202 \text { TBI; } \\
56 \text { mTBI } 56 \\
104 \text { moderate/severe TBI } \\
42 \text { unknown } \\
73 \text { HC }\end{array}$ & $\begin{array}{l}\text { At least } 12 \\
\text { months after injury, } \\
15 \text { (12) years }\end{array}$ & $\begin{array}{l}\text { TBI } 47(12) \\
\text { HC } 41(12)\end{array}$ & $\begin{array}{l}\text { TBI } 109 / 93 \\
\text { HC } 28 / 45\end{array}$ & $\begin{array}{l}\text { Cambridge } \\
\text { Neuropsychological } \\
\text { Test Automated } \\
\text { Battery, repeated } 3 \\
\text { times. } \\
\text { The second and third } \\
\text { administrations of the } \\
\text { battery separated by } 2 \\
\text { h of interviews and } \\
\text { administration of } \\
\text { self-report measures. } \\
\text { Global Fatigue Index } \\
\text { State-fatigue rating on } \\
\text { Likert scale at the } \\
\text { beginning and end. }\end{array}$ & $\begin{array}{l}\text { TBI group performed } \\
\text { worse at all } 3 \text { time points } \\
(p<0.05) \text {. } \\
\text { Time by group interaction } \\
\text { indicated improved speed } \\
\text { between T1 and T2 for } \\
\text { controls }(p=0.04) \text {. } \\
\text { TBI group did not vary } \\
\text { significantly across all } \\
3 \text { trials. } \\
\text { State-fatigue was related to } \\
\text { speed subscale at all trial } \\
(p<0.05) .\end{array}$ \\
\hline $\begin{array}{l}\text { Azouvi et al., } \\
2004[60]\end{array}$ & $\begin{array}{l}43 \text { severe TBI } \\
42 \mathrm{HC}\end{array}$ & 10 (11) months & $\begin{array}{l}\text { TBI } 26 \text { (8) } \\
\text { HC } 27 \text { (9) }\end{array}$ & $\begin{array}{l}32 / 11 \\
\text { HC matched }\end{array}$ & $\begin{array}{l}\text { Visual-go-no-go task } \\
\text { VAS-fatigue }\end{array}$ & $\begin{array}{l}\text { A worsening of } \\
\text { performance after severe } \\
\text { TBI on a dual-task with } \\
\text { load on working memory } \\
\text { and/or executive control } \\
\text { (interaction } p>0.05 \text { ). }\end{array}$ \\
\hline $\begin{array}{l}\text { Belmont, Agar, \& } \\
\text { Azouvi, } 2009 \text { [61] }\end{array}$ & $\begin{array}{l}27 \text { severe TBI } \\
26 \mathrm{HC}\end{array}$ & 9 (5) months & $\begin{array}{l}\text { TBI } 32(9) \\
\text { HC } 32(10)\end{array}$ & $\begin{array}{l}\text { TBI } 21 / 6 \\
\text { HC 20/6 }\end{array}$ & $\begin{array}{l}\text { Go/No GO (Selective } \\
\text { attention task) } \\
\text { During the break } \\
\text { between the } 2 \text { parts of } \\
\text { the task (T1), and at } \\
\text { the end of the second } \\
\text { part of the task. (T2) } \\
\text { FSS } \\
\text { VAS-fatigue }\end{array}$ & $\begin{array}{l}\text { Patients with severe TBI } \\
\text { with a high baseline } \\
\text { fatigue performed less well } \\
\text { on the selective attention } \\
\text { test with a longer reaction } \\
\text { time }(p<0.001) \text { and more } \\
\text { omissions compared to } \\
\text { controls }(p<0.001) \text {. } \\
\text { Significant correlations } \\
\text { were found in the TBI } \\
\text { group between attention } \\
\text { performance, mental effort, } \\
\text { and subjective fatigue } \\
(p<0.05) \text {. }\end{array}$ \\
\hline
\end{tabular}


Table 1. Cont.

\begin{tabular}{|c|c|c|c|c|c|c|}
\hline Reference & $\begin{array}{c}\text { Number of Participants } \\
\text { and } \\
\text { Injury Severity }\end{array}$ & Time Since Injury & $\begin{array}{c}\text { Age } \\
\text { Year Mean } \\
\text { (sd) }\end{array}$ & $\begin{array}{c}\text { Sex } \\
\text { (Males/ } \\
\text { Females) }\end{array}$ & $\begin{array}{l}\text { Cognitive Test } \\
\text { Fatigue Measure }\end{array}$ & Results \\
\hline $\begin{array}{l}\text { Berginström et al., } \\
2018 \text { [25] }\end{array}$ & $\begin{array}{l}57 \mathrm{TBI} ; \\
7 \text { sever } \\
10 \text { moderate } \\
40 \text { mild } \\
27 \mathrm{HC}\end{array}$ & $9(7)$ years & $\begin{array}{l}\text { TBI } 42(13) \\
\text { HC } 38(12)\end{array}$ & $\begin{array}{l}\text { TBI } 31 / 26 \\
\text { fem } \\
\text { HC } 14 / 13\end{array}$ & $\begin{array}{l}27 \text { min modified } \\
\text { SDMT } \\
\text { FSS } \\
\text { MFS }\end{array}$ & $\begin{array}{l}\text { TBI reported increased } \\
\text { fatigue after the test } \\
\text { session having a slower } \\
\text { reaction time compared to } \\
\text { controls }(p<0.001) \text {. } \\
\text { Reaction time remained } \\
\text { constant for both groups } \\
\text { during the session. }\end{array}$ \\
\hline $\begin{array}{l}\text { Borgaro, Gierok, } \\
\text { Caples, \& } \\
\text { Kwasnica, } 2004 \\
\text { [68] }\end{array}$ & $\begin{array}{l}47 \text { TBI; } \\
18 \text { severe TBI } \\
18 \text { moderate TBI } \\
11 \text { mTBI } \\
\text { HC } 30\end{array}$ & 24 (17) days & $\begin{array}{l}\text { TBI } 36(16) \\
\text { HC } 36(20)\end{array}$ & & $\begin{array}{l}\text { BNI screening for } \\
\text { cognitive function } \\
\text { BNI fatigue scale }\end{array}$ & $\begin{array}{l}\text { TBI reported significantly } \\
\text { greater levels of fatigue } \\
\text { compared to controls. } \\
\text { Fatigue was unrelated to } \\
\text { injury severity, number of } \\
\text { days from injury to } \\
\text { assessment, cognitive } \\
\text { impairment, and gender. }\end{array}$ \\
\hline $\begin{array}{l}\text { Johansson et al., } \\
2009 \text { [34] }\end{array}$ & $\begin{array}{l}60 \text { TBI; } \\
14 \mathrm{mTBI} \text { fulltime work } \\
\text { (FW) } \\
34 \mathrm{mTBI} \\
12 \text { TBI moderate/severe } \\
40 \mathrm{HC}\end{array}$ & $\begin{array}{l}\text { Years } \\
\text { mTBI FW } 6 \text { (2) } \\
\text { mTBI (7) (1) } \\
\text { TBI } 11 \text { (2) }\end{array}$ & $\begin{array}{l}\text { mTBI FW } 45 \\
(2) \\
\text { mTBI } 52(1) \\
\text { TBI } 42(4) \\
\text { HC } 42(1)\end{array}$ & $\begin{array}{l}\text { mTBI FW } \\
6 / 8 \\
\text { mTBI } 7 / 25 \\
\text { TBI } 5 / 7 \\
\text { HC } 16 / 24\end{array}$ & $\begin{array}{l}\text { Digit symbol-coding, } \\
\text { reading speed, trail } \\
\text { making test, digit } \\
\text { span, spatial span, } \\
\text { verbal fluency. } \\
\text { MFS }\end{array}$ & $\begin{array}{l}\text { Reduced processing speed } \\
\text { (digit symbol-coding, } \\
\text { reading speed, trail } \\
\text { making test) correlated } \\
\text { with increased mental } \\
\text { fatigue }(p<0.05) \text {. }\end{array}$ \\
\hline $\begin{array}{l}\text { Johansson \& } \\
\text { Rönnbäck, } 2015 \\
\text { [69] }\end{array}$ & $\begin{array}{l}76 \mathrm{mTBI} \\
45 \mathrm{HC}\end{array}$ & 9 (8) years & $\begin{array}{l}\operatorname{mTBI} 43(12) \\
\text { HC } 41 \text { (12) }\end{array}$ & $\begin{array}{l}\operatorname{mTBI} 30 / 46 \\
\mathrm{HC} 15 / 30\end{array}$ & $\begin{array}{l}\text { A five-minute } \\
\text { computer test with } \\
\text { five repetitions, } \\
\text { including task } \\
\text { repetitions with } \\
\text { simultaneous load on } \\
\text { processing speed and } \\
\text { working memory. } \\
\text { MFS }\end{array}$ & $\begin{array}{l}\text { During the test, the } \\
\text { controls became } \\
\text { significantly faster, while } \\
\text { this was not found for the } \\
\text { mTBI group who remained } \\
\text { on a similar slower speed } \\
(p<0.05) \text {. }\end{array}$ \\
\hline $\begin{array}{l}\text { Möller, Nygren } \\
\text { de Boussard, } \\
\text { Oldenburg, \& } \\
\text { Bartfai, 2014 [64] }\end{array}$ & $\begin{array}{l}24 \mathrm{mTBI} \\
31 \mathrm{HC}\end{array}$ & & $\begin{array}{l}\text { mTBI } 36 \\
\text { HC } 37\end{array}$ & $\begin{array}{l}\text { mTBI } 12 / 12 \\
\text { HC } 13 / 18\end{array}$ & $\begin{array}{l}\text { Digit Symbol } \\
\text { Substitution Test } \\
\text { Executive functions } \\
\text { (Color Word Test) } \\
\text { Attention (Ruff) } \\
\text { FSS }\end{array}$ & $\begin{array}{l}\text { Decreased performance } \\
\text { (fatigability) was reported } \\
\text { for the mTBI group who } \\
\text { reported more fatigue than } \\
\text { controls in more } \\
\text { demanding cognitive tests } \\
\text { with simultaneous } \\
\text { activation of several } \\
\text { cognitive domains and } \\
\text { executive functions } \\
\text { ( } p<0.01) \text {. Fatigability was } \\
\text { not found to be present for } \\
\text { less demanding tests } \\
\text { involving simple } \\
\text { automatic attention. }\end{array}$ \\
\hline $\begin{array}{l}\text { Möller et al., } 2017 \\
\text { [26] }\end{array}$ & $\begin{array}{l}10 \mathrm{mTBI} \\
10 \mathrm{HC}\end{array}$ & $\begin{array}{l}\text { At least } 6 \text { months } \\
\text { after }\end{array}$ & $\begin{array}{l}\operatorname{mTBI} 38(11) \\
\text { HC } 37(11)\end{array}$ & $\begin{array}{l}\operatorname{mTBI} 5 / 5 \\
\mathrm{HC} 5 / 5\end{array}$ & $\begin{array}{l}\text { Psychomotor } \\
\text { vigilance task } \\
\text { FSS }\end{array}$ & $\begin{array}{l}\text { Reaction time was longer } \\
\text { and increased during a } \\
\text { 20-min vigilance task for } \\
\text { mTBI group, while the } \\
\text { reaction time remained } \\
\text { stable for the controls. The } \\
\text { results showed that RT for } \\
\text { patients and controls } \\
\text { differed significantly } \\
(p=0.005) \text {. }\end{array}$ \\
\hline $\begin{array}{l}\text { Möller, } \\
\text { Johansson, } \\
\text { Matuseviciene, } \\
\text { Pansell, \& } \\
\text { Nygren } \\
\text { Deboussard, } 2019 \\
\text { [67] }\end{array}$ & $\begin{array}{l}15 \mathrm{mTBI} \\
15 \mathrm{OC}\end{array}$ & 7-10 days & $\begin{array}{l}\operatorname{mTBI} 25(6) \\
\text { OC } 28(7)\end{array}$ & $\begin{array}{l}\operatorname{mTBI} 7 / 8 \\
\text { OC } 11 / 4\end{array}$ & $\begin{array}{l}\text { Saccade function } \\
\text { Digit Symbol } \\
\text { Substitution Test } \\
\text { (DSST) } \\
\text { The Ruff } 2 \text { \& } 7 \\
\text { Selective Attention } \\
\text { Test } \\
\text { FSS } \\
\text { RPQ was used for } \\
\text { self-rated change in } \\
\text { fatigue pre- post } \\
\text { injury }\end{array}$ & $\begin{array}{l}\text { mTBI scored higher fatigue } \\
\text { on RPQ compared with } \\
\text { controls }(p=0.023 \text { ) and } \\
\text { cognitive fatigability } \\
\text { (DSST, } p=0.024) \text {. FSS did } \\
\text { not differ significantly } \\
\text { between patients and OC. } \\
\text { Acquired fatigue } \\
\text { correlated to slower } \\
\text { prosaccade performance } \\
\text { (RPQ } p=0.006) \text {. FSS } \\
\text { correlated to slower and } \\
\text { unstable antisaccade } \\
\text { performance }(p=0.019 \text { ). } \\
\text { The more fatigability the } \\
\text { more errors on the Ruff } 2 \& \\
7 \text { subtask-controlled } \\
\text { attention }(p=0.022) \text {. }\end{array}$ \\
\hline
\end{tabular}


Table 1. Cont.

\begin{tabular}{|c|c|c|c|c|c|c|}
\hline Reference & $\begin{array}{c}\text { Number of Participants } \\
\text { and } \\
\text { Injury Severity }\end{array}$ & Time Since Injury & $\begin{array}{c}\text { Age } \\
\text { Year Mean (sd) }\end{array}$ & $\begin{array}{c}\text { Sex } \\
\text { (Males/ } \\
\text { Females) }\end{array}$ & $\begin{array}{l}\text { Cognitive Test } \\
\text { Fatigue Measure }\end{array}$ & Results \\
\hline $\begin{array}{l}\text { Rau et al., } 2017 \\
\text { [70] }\end{array}$ & $\begin{array}{l}17 \mathrm{mTBI} \\
17 \mathrm{HC}\end{array}$ & 9 (3) months & $\begin{array}{l}\operatorname{mTBI} 30(5) \\
\text { HC } 31(5)\end{array}$ & $\begin{array}{l}\operatorname{mTBI} 10 / 7 \\
\text { HC } 8 / 9\end{array}$ & $\begin{array}{l}\text { Repetition ( } 3 \text { times) of } \\
\text { a working memory } \\
\text { and wordlist memory } \\
\text { test } \\
\text { No fatigue measure, } \\
\text { but indirectly suffered } \\
\text { from post-concussion. }\end{array}$ & $\begin{array}{l}\text { No interaction for } \\
\text { group by time, but } \\
\text { significant main } \\
\text { effects for time } \\
(p=0.04) \text { and group } \\
(p<0.001) \text {. The mTBI } \\
\text { performed worse from } \\
\text { time points } 1 \text { to } 3 \\
(p=0.02) \text {. Time } 3 \\
\text { showed significant } \\
\text { difference between the } \\
\text { groups }(p<0.001) \text {. }\end{array}$ \\
\hline $\begin{array}{l}\text { Skau et al., } 2019 \\
\text { [24] }\end{array}$ & $\begin{array}{l}20 \mathrm{mTBI} \\
20 \mathrm{HC}\end{array}$ & 28 (21) months & $\begin{array}{l}\text { mTBI } 42(10) \\
\text { HC } 39(11)\end{array}$ & $\begin{array}{l}\operatorname{mTBI} 7 / 13 \\
\mathrm{HC} 8 / 12\end{array}$ & $\begin{array}{l}\text { 2.5-h test session } \\
\text { One repetition } \\
\text { Stroop-Simon } \\
\text { Digit symbol coding, } \\
\text { Digit span, } \\
\text { Symbol search } \\
\text { MFS } \\
\text { VAS-energy }\end{array}$ & $\begin{array}{l}\text { A significant reduced } \\
\text { mental energy for the } \\
\text { mTBI after } 2.5 \mathrm{~h} \\
(p<0.01) \text {. The controls } \\
\text { improved the second } \\
\text { time on Digit Symbol } \\
\text { Coding ( } p<0.05) \text {, } \\
\text { while the mTBI } \\
\text { remained on a similar } \\
\text { lower level. }\end{array}$ \\
\hline $\begin{array}{l}\text { Ziino \& Ponsford, } \\
\text { 2006a [62] }\end{array}$ & $\begin{array}{l}49 \text { mild to severe TBI } \\
46 \mathrm{HC}\end{array}$ & 240 (222) days & $\begin{array}{l}\text { TBI } 35 \text { (13) } \\
\text { HC } 34(10)\end{array}$ & $\begin{array}{l}\text { TBI } 63 \% \text { males } \\
\text { HC } 61 \% \text { males }\end{array}$ & $\begin{array}{l}\text { Complex selective } \\
\text { attention task } \\
\text { FSS } \\
\text { VAS-fatigue }\end{array}$ & $\begin{array}{l}\text { Slower performance } \\
\text { on a C-SAT for TBI } \\
\text { group comparison } \\
\text { with controls } \\
(p<0.001) \text {, and it was } \\
\text { associated with } \\
\text { fatigue for mild to } \\
\text { severe TBI }(p<0.05) \text {. }\end{array}$ \\
\hline $\begin{array}{l}\text { Ziino \& Ponsford, } \\
\text { 2006b [63] }\end{array}$ & $\begin{array}{l}46 \text { mild to severe TBI } \\
46 \mathrm{HC}\end{array}$ & 240 (222) days & $\begin{array}{l}\text { TBI } 35(13) \\
\text { HC } 34(10)\end{array}$ & & $\begin{array}{l}\text { A vigilance test with a } \\
\text { duration of } 45 \mathrm{~min} \\
\text { FSS } \\
\text { VAS-fatigue }\end{array}$ & $\begin{array}{l}\text { The TBI group was } \\
\text { slower and remained } \\
\text { at a similar slower } \\
\text { speed throughout the } \\
\text { duration of the task } \\
(p<0.001) \text {. Higher } \\
\text { state-fatigue was } \\
\text { associated with more } \\
\text { omissions for TBI. A } \\
\text { subgroup of TBI } \\
\text { showed a decline in } \\
\text { performance } \\
(p<0.05) \text {. }\end{array}$ \\
\hline
\end{tabular}

Abbreviations: sd (standard deviation), HC (healthy controls), OC (orthopedic controls), SDMT (Symbol Digit Modality Test), BNI (Barrow Neurological Institute), MFI (Multidimensional Fatigue Inventory), FSS (Fatigue Severity Scale), MFS (Mental Fatigue Scale), VAS (Visual Analogue Scale), RPQ (Rivermead Post-Concussion Symptoms Questionnaire), mTBI (mild Traumatic Brain Injury).

When combining endurance and cognitive function among people suffering from mental fatigue after a TBI, no change was found when tests were repeated, while improvement was achieved for healthy controls [24,59,68,69]. A 30-min computerized neuropsychological test battery using the Cambridge Neuropsychological Test Automated Battery with three repetitions, with the second and third repetition separated by approximately $2 \mathrm{~h}$ of interviews and self-report measures, was compared between TBI and control groups. Fatigue was associated with poor performance in speed for the TBI group, but this was not associated with an objective decline in cognitive tests. On the contrary, improvement on speed and accuracy was found in the control group [59]. A five-minute computer test with five repetitions, including task repetitions with simultaneous load on processing speed and working memory, showed an overall slower speed for the mTBI group. During the test, the controls became significantly faster when they repeated the task, while this was not found for the mTBI group, who remained on a similar slower speed [69]. After a 2.5-h test session, there was no change in test performance for the mTBI group (processing speed, working memory, attention), but a significantly reduced mental energy compared to the controls was reported after the test session. In contrast, the control group improved their processing speed (Digit symbol coding, WAIS-IV) when the test was repeated [24]. Repetition (three times) of a working memory and wordlist memory test, with 60 sec. rest between trials, revealed no difference between control and mTBI groups after the first trial. However, a significant difference was reported between groups in working memory after trial three, 
and a significant difference was also found between groups for word-list learning after trials two and three, with the controls performing better with a greater improvement in word-list learning. It was suggested that repetition of tests can be useful for distinguishing cognitive fatigue [70].

In summary, several studies have demonstrated that fatigue is related to information processing speed, attention, and memory. More demanding cognitive tests are suggested to be related to fatigue, but also automatic and simple cognitive tests are suggested to be related to fatigue. This is not a simple relationship, and results can vary depending on which tests and study design are used. However, in no case has improved cognitive function been reported for those suffering from fatigue after an mTBI or TBI. The measurement of endurance, using the repetition of tests, is a promising means of assessment. Fatigability, namely a worsening of performance, is not an accurate means of assessment. The preferred method of assessment is altered performance over time or after repetition of tasks compared to controls.

It can be argued whether the injury per se is related to impaired cognitive function and, to a lesser extent, to mental fatigue. However, from a study including a recovered mTBI group, no difference was found in mental fatigue and reaction time between those who had recovered and the controls, while a significantly higher MFS and a slower reaction time were reported for those with persistent mTBI [71].

\subsection{Brain Imaging in Relation to TBI and Fatigue}

Brain imaging as an assessment tool for fatigue after TBI is in its infancy. A few recent studies have shown promising results (Table 2). For participants with a moderate to severe TBI, increased brain activity was reported during a one-hour scanning, while controls showed decreased brain activity in a processing speed task, Symbol Digit Modalities Test -(SDMT), measured with functional magnetic resonance imaging (fMRI). Increased brain activity was suggested to represent increased effort and fatigue. No assessment of trait- or state-fatigue was performed [27]. In contrast, when the same processing speed test (SDMT) was performed, lower brain activity (fMRI) in the basal ganglia, primarily the caudate nucleus, thalamus, and anterior insula, was reported for the TBI group (ranging from mild to severe) compared to controls. The brain activity decreased across the 27-min test session for the controls, whereas the TBI group remained on a similar lower activity level and reported an increased state-fatigue after the session compared to controls [25]. A group of moderate to severe TBI patients reported increased fatigue during the test session compared to controls. Brain activation showed a positive relation between fatigue and fMRI for the more advanced 2-back working memory task and a negative relation for the simpler task (0-back task). Fatigue in relation to brain activation was related to the caudate nucleus [28]. Using functional near-infrared spectroscopy (fNIRS) to measure brain activity during a $2.5 \mathrm{~h}$ session, a lower event-related oxygenated haemoglobin $(\mathrm{oxy}-\mathrm{Hb})$ concentration was detected in the frontal cortex for the mTBI group, all of whom were suffering from mental fatigue compared to controls. The Stroop-Simon test was repeated once, and other cognitive tests and assessments scales were performed between these. The event activation remained on a similar lower level for the mTBI group and on a similar albeit higher level for the control group. An interaction was found, with the mTBI group having a similar lower oxy- $\mathrm{Hb}$ concentration for both congruent and incongruent trials, whereas the controls had a higher concentration of oxy- $\mathrm{Hb}$ in the more demanding incongruent trial compared to the congruent trial. Higher mental fatigue correlated with lower oxy-HB [24]. A group suffering from fatigue after mTBI used different brain networks compared to healthy controls during a 20-min vigilance task. There was a correlation between fatigue and functional connectivity in the thalamus and middle frontal cortex and changes in regional cerebral blood flow ( $\mathrm{rCBF}$ ). $\mathrm{rCBF}$ was related to fatigability with increased $\mathrm{rCBF}$ in the right middle frontal gyrus. Self-rated fatigue was related to increased $\mathrm{rCBF}$ in the left medial frontal and anterior cingulate gyri and decreases of $\mathrm{rCBF}$ in a frontal/thalamic network $[26,29]$. To evaluate mental fatigue associated with $\mathrm{mTBI}$ in acute and chronic 
phases, a 20-min psychomotor vigilance test was related to arterial spin labeling-fMRI. Sustained attention was impaired in mTBI patients both in acute and in chronic phases compared to controls, and with worse performance in the acute phase [72]. Brain activation associated with effort and fatigue did not differentiate the mTBI and controls, while functional connectivity did. Connectivity between the left anterior insula, rostral anterior cingulate cortex, and right-sided inferior frontal regions correlated with effort level and state-fatigue in mTBI participants. These connections also correlated with effort level in the controls. Left insula and superior medial frontal gyrus correlated with fatigue. The authors propose a complex link between effort and fatigue and that it may be related to an inefficient neuronal system [73]. Activation assessed with rCBF within the cerebellar cortex correlated with PASAT, measuring information processing and sustained and divided attention in controls, while mTBI participants with self-reported cognitive fatigue showed significant correlations in the inferior frontal and superior temporal cortices [74]. MRI revealed grey matter (GM) and white matter (WM) brain lesions in a TBI group, but fatigue was not found to be related to brain lesions [75]. White matter hyperintensity lesions (WMH) were more common in mild-severe TBI patients compared to controls, but WMH lesions were not related to cognitive tests. Increased WMH lesions correlated with reduced fatigue, but the relation to fatigue was reported to be weak [76]. An mTBI group had a larger number of low diffusion tensor imaging measures (fractional anisotropy values) and more post-concussion symptoms (fatigue not measured separately but included in the PCS) compared to controls. No difference in neurocognitive tests was found [77]. In mild to moderate TBI participants, compared to controls, decreased white matter integrity of the left anterior internal capsule was associated with a greater level of fatigue [78]. A higher rating of fatigue in an mTBI group was associated with decreased right and left thalamic volumes [79]. From the Vietnam Head Injury Study registry, individuals with penetrating brain injuries (PBI) were assessed with a self-report questionnaire and computed tomography (CT) scans. Lesion location and volume were evaluated. PBI patients were divided into three groups according to lesion location: a nonfrontal lesion group, a ventromedial prefrontal cortex (vmPFC) lesion group, and a dorso/lateral prefrontal cortex group. Fatigue scores were compared between the three PBI groups and the healthy controls. Individuals with PBI with vmPFC lesion were significantly more fatigued than the other groups as well as the healthy controls. VmPFC volume correlated with fatigue scores showing that the larger the lesion volume, the higher the fatigue scores [80].

Table 2. Research relating fatigue in adults after TBI to brain imaging techniques. Summary of study characteristics, including details on study sample, methods, and results.

\begin{tabular}{|c|c|c|c|c|c|c|}
\hline Reference & $\begin{array}{c}\text { Number of } \\
\text { Participants } \\
\text { Injury Severity }\end{array}$ & Time Since Injury & $\begin{array}{c}\text { Age } \\
\text { Year (sd) }\end{array}$ & $\begin{array}{c}\text { Sex } \\
\text { (Males/ } \\
\text { Females) }\end{array}$ & $\begin{array}{l}\text { Brain Imaging } \\
\text { Fatigue Measure }\end{array}$ & Results \\
\hline $\begin{array}{l}\text { Berginström et al., } \\
2018 \text { [25] }\end{array}$ & $\begin{array}{l}57 ; \text { TBI } \\
40 \text { mild } \\
10 \text { moderate } \\
7 \text { severe } \\
27 \mathrm{HC}\end{array}$ & 9 (7) years & $\begin{array}{l}\text { TBI } 42(13) \\
\text { HC } 38 \text { (12) }\end{array}$ & $\begin{array}{l}\text { TBI } 31 / 26 \\
\text { HC } 14 / 13\end{array}$ & $\begin{array}{l}\text { fMRI, modified } \\
\text { SDMT } \\
\text { FSS } \\
\text { MFS } \\
\text { VAS-fatigue }\end{array}$ & $\begin{array}{l}\text { Lower brain activity (fMRI) in } \\
\text { basal ganglia, primarily the } \\
\text { caudate nucleus, thalamus, and } \\
\text { anterior insula for the TBI group } \\
\text { compared to controls (all } p<0.05 \text { ). } \\
\text { The brain activity decreased } \\
\text { across the } 27 \text {-min test session for } \\
\text { the controls, whereas the TBI } \\
\text { group remained on a similar } \\
\text { lower activity level. Increased } \\
\text { state-fatigue after the session } \\
\text { compared to controls was } \\
\text { reported }(p<0.01) \text {. }\end{array}$ \\
\hline $\begin{array}{l}\text { Berginström, } \\
\text { Nordström, Nyberg, } \\
\text { \& Nordström, } 2020 \\
{[76]}\end{array}$ & $\begin{array}{l}59 \text { TBI; } \\
40 \text { mild } \\
11 \text { moderate } \\
8 \text { severe } \\
27 \mathrm{HC}\end{array}$ & $\begin{array}{l}\text { TBI } 42(9) \\
\text { HC } 38 \text { (12) }\end{array}$ & 9 (7) years & $\begin{array}{l}\text { TBI } 32 / 27 \\
\text { HC } 14 / 13\end{array}$ & $\begin{array}{l}\text { WM hyperintensity } \\
\text { lesions } \\
\text { FSS } \\
\text { MFS }\end{array}$ & $\begin{array}{l}\text { WMH lesions were more common } \\
\text { in TBI compared to controls. } \\
\text { WMH lesions were not related to } \\
\text { cognitive tests. } \\
\text { Increased WMH lesions correlated } \\
\text { with reduced fatigue }(p=0.026) \text {. }\end{array}$ \\
\hline
\end{tabular}


Table 2. Cont.

\begin{tabular}{|c|c|c|c|c|c|c|}
\hline Reference & $\begin{array}{c}\text { Number of } \\
\text { Participants } \\
\text { Injury Severity }\end{array}$ & Time Since Injury & $\begin{array}{c}\text { Age } \\
\text { Year (sd) }\end{array}$ & $\begin{array}{c}\text { Sex } \\
\text { (Males/ } \\
\text { Females) }\end{array}$ & $\begin{array}{l}\text { Brain Imaging } \\
\text { Fatigue Measure }\end{array}$ & Results \\
\hline $\begin{array}{l}\text { Clark et al., } 2017 \\
\text { [78] }\end{array}$ & $\begin{array}{l}59 \text { TBI } \\
\text { mild-moderate } \\
\text { HC } 25\end{array}$ & 64 (34) months & $\begin{array}{l}\text { TBI } 33(6) \\
\text { HC } 34(8)\end{array}$ & $\begin{array}{l}88 \% \text { males } \\
72 \% \text { males }\end{array}$ & $\begin{array}{l}\text { DTI } \\
\text { MFIS }\end{array}$ & $\begin{array}{l}\text { Decreased white matter } \\
\text { microstructural integrity of left } \\
\text { anterior internal capsule }(p=0.02) \\
\text { involved in basal ganglia circuitry } \\
\text { in mTBI compared to HC, and this } \\
\text { was associated with greater level } \\
\text { of fatigue }(p=0.01) \text {. }\end{array}$ \\
\hline
\end{tabular}

Greater levels of fatigue were associated with decreased right $(p=0.026)$ and left $(p=0.046)$

\begin{tabular}{|c|c|c|c|c|c|}
\hline $\begin{array}{l}\text { Clark et al., } 2018 \\
\text { [79] }\end{array}$ & $63 \mathrm{mTBI}$ & 64 (43) months & $32(6)$ & $87 \%$ males & $\begin{array}{l}\text { MRI } \\
\text { Thalamic volume } \\
\text { MFIS }\end{array}$ \\
\hline
\end{tabular}

Functional connectivity was influenced by PVT task with a significant difference between mTBI and HC $(p<0.05)$ in

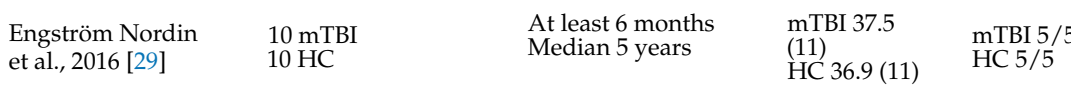

Psychomotor vigilance task (PVT) thalamus and middle frontal adapted to MRI VAS-fatigue

more extensive functional and stronger functional connectivity network in thalamus connectivity in medial frontal cortex both before and after the PVT task.

In all 4 trials, mild TBI had lower PASAT scores $(p<0.05)$ A different pattern of $\mathrm{rCBF}$
rCBF SPECT PASAT (information processing and sustained and divided attention) Persistent cognitive complaints, specifically the complaint of cognitive fatigue between $\mathrm{mTBI}$ and $\mathrm{HC}(p<0.05)$.

Less activation for $\mathrm{mTBI}$ in the cerebellum and more activation in the prefrontal cortex. mTBI showed dynamic changes in supratentorial rCBF during PASAT, with larger areas of activation bilaterally in the dorsolateral prefrontal cortex and larger areas of suppression in the occipital and parietal cortices.

TBI group increased activity in the middle frontal gyrus, superior parietal cortex, basal ganglia, and anterior cingulate.

Controls decreased activity over time $(p<0.005)$ TBI had slower reaction during all 3 times $(p<0.05)$.

PVT was related to arterial spin labeling-fMRI. Sustained attention was impaired in mTBI patients both in acute and in chronic phases comapred to controls, and with worse performance in the acute phase.

A significant interaction effect between mTBI and HC in several T, fatigability at the end of was related to increased rCBF in the right middle frontal gyrus. Self-rated fatigue was related to increased $\mathrm{rCBF}$ in left medial frontal and anterior cingulate gyri and decreases of $\mathrm{rCBF}$ in a frontal/thalamic network.

97 penetrating brain

injuries

Divided into; 17 ventromedial

Pardini et al., 2010 prefrontal cortex [80]

$\begin{array}{lll}\text { At least } 6 \text { months } & \text { mTBI } 38(11) & \text { mTBI } 5 / 5\end{array}$
HC 37 (11) HC 5/5
rCBF SPEC
Psychomoto FSS
Individuals with PBI with vmPFC lesion were significantly more fatigued than the other groups as well as the healthy controls $(p=0.013)$. VmPFC volume correlated with fatigue scores $(p=0.0053)$, the larger the lesion volume, the higher the fatigue scores. 
Table 2. Cont

\begin{tabular}{|c|c|c|c|c|c|c|}
\hline Reference & $\begin{array}{c}\text { Number of } \\
\text { Participants } \\
\text { Injury Severity }\end{array}$ & Time Since Injury & $\begin{array}{c}\text { Age } \\
\text { Year (sd) }\end{array}$ & $\begin{array}{c}\text { Sex } \\
\text { (Males/ } \\
\text { Females) }\end{array}$ & $\begin{array}{l}\text { Brain Imaging } \\
\text { Fatigue Measure }\end{array}$ & Results \\
\hline $\begin{array}{l}\text { Ramage, Tate, New, } \\
\text { Lewis, \& Robin, } \\
2019 \text { [73] }\end{array}$ & $\begin{array}{l}60 \mathrm{mTBI} \\
42 \mathrm{OC}\end{array}$ & $\begin{array}{l}\text { At least } 60 \text { days } \\
\text { prior to assessment } \\
292 \text { (176) days }\end{array}$ & $\begin{array}{l}\operatorname{mTBI} 36(8) \\
\text { OC } 33(10)\end{array}$ & $\begin{array}{l}\operatorname{mTBI} 53 / 7 \\
\text { OC } 40 / 2\end{array}$ & $\begin{array}{l}\text { fMRI } \\
\text { Functional } \\
\text { Connectivity } \\
\text { Constant Effort Task } \\
\text { FSS }\end{array}$ & $\begin{array}{l}\text { Brain activation associated with } \\
\text { effort and fatigue did not } \\
\text { differentiate the mTBI and } \\
\text { controls, while functional } \\
\text { connectivity did. FSS correlated } \\
\text { with functional connectivity } \\
\text { between the left insula and the } \\
\text { dorsal anterior cingulate cortex } \\
(p<0.01) \text {, the left insula and the } \\
\text { right inferior frontal gyrus } \\
(p<0.05) \text {, and the dorsal anterior } \\
\text { cingulate cortex and the right } \\
\text { inferior frontal gyrus ( } p<0.05) \\
\text { medial frontal gyrus correlated } \\
\text { with FSS, all during the first half } \\
\text { of the } 75 \% \text { effort level. }\end{array}$ \\
\hline $\begin{array}{l}\text { Schönberger et al., } \\
2017 \text { [75] }\end{array}$ & $\begin{array}{l}53 \text { TBI } \\
\text { mild to severe, most } \\
\text { moderate or severe } \\
36 \text { subgroup } \\
\text { vigilance test }\end{array}$ & 2 (1) years & $38(14)$ & $77 \%$ male & $\begin{array}{l}\text { MRI total brain } \\
\text { volume, and lesions; } \\
\text { GM and WM } \\
\text { separately as well } \\
\text { as combined. } \\
\text { Vigilance task } \\
\text { FSS }\end{array}$ & $\begin{array}{l}\text { MRI revealed GM and WM brain } \\
\text { lesions but fatigue was not related } \\
\text { to brain lesions. }\end{array}$ \\
\hline $\begin{array}{l}\text { Skau, } \\
\text { Bunketorp-Käll, } \\
\text { Kuhn, \& Johansson, } \\
2019 \text { [24] }\end{array}$ & $\begin{array}{l}20 \mathrm{mTBI} \\
20 \mathrm{HC}\end{array}$ & 28 (21) months & $\begin{array}{l}\text { mTBI } 42(10) \\
\text { HC } 39(11)\end{array}$ & $\begin{array}{l}\operatorname{mTBI} 7 / 13 \\
\mathrm{HC} 8 / 12\end{array}$ & $\begin{array}{l}\text { fNIRS, modified } \\
\text { Stroop-Simon, one } \\
\text { repetition } \\
\text { MFS } \\
\text { VAS- energy }\end{array}$ & $\begin{array}{l}\text { Lower event-related oxygenated } \\
\text { hemoglobin (oxy-Hb) } \\
\text { concentration in the frontal cortex } \\
\text { for the mTBI group, compared to } \\
\text { controls }(p<0.05) \text {. No time effect. } \\
\text { An interaction }(p<0.05) \text { was } \\
\text { found, with the mTBI group } \\
\text { having a similar lower oxy-Hb } \\
\text { concentration for both congruent } \\
\text { and incongruent trials, whereas } \\
\text { the controls had a higher } \\
\text { concentration of oxy-Hb in the } \\
\text { more demanding incongruent } \\
\text { trial compared to the congruent } \\
\text { trial. Higher MFS correlated with } \\
\text { lower oxy-HB }(p<0.05) \text {. }\end{array}$ \\
\hline $\begin{array}{l}\text { Wylie et al., } 2017 \\
\text { [28] }\end{array}$ & $\begin{array}{l}22 ; \\
20 \text { TBI moderate to } \\
\text { severe, } \\
2 \text { complicated mTBI } \\
20 \mathrm{HC}\end{array}$ & 80 (51) months & $\begin{array}{l}\text { TBI } 41 \text { (13) } \\
\text { HC } 38(11)\end{array}$ & $\begin{array}{l}\text { TBI } 14 / 8 \\
\text { HC } 8 / 14\end{array}$ & $\begin{array}{l}\text { fMRI } \\
\text { Four blocks of } \\
\text { working memory, } \\
\text { 2-back task } \\
\text { (difficult), and } \\
\text { 0-back } \\
\text { speed } \\
\text { VAS-fatigue }\end{array}$ & $\begin{array}{l}\text { TBI group was slower in response } \\
\text { time }(p<0.001) \text {. Fatigue } \\
\text { interacted with task in several } \\
\text { areas. Negative correlation } \\
\text { between reaction time and fatigue } \\
(p=0.08) \\
\text { TBI; correlation between fatigue } \\
\text { and activation for 2-back and } \\
0 \text {-back were weak } \\
\text { (coefficient }=0.0003 \text { ). HC; there } \\
\text { was a positive correlation } \\
\text { between fatigue and brain } \\
\text { activation for difficult } 2 \text {-back task } \\
\text { (coefficient }=0.0035) \text {, and negative } \\
\text { for the } 0 \text {-back task } \\
\text { (coefficient }=-0.0013 \text { ). Fatigue in } \\
\text { relation to brain activation was } \\
\text { related to caudate nucleus. }\end{array}$ \\
\hline $\begin{array}{l}\text { Wäljas et al., } 2014 \\
\text { [77] }\end{array}$ & $\begin{array}{l}48 \mathrm{mTBI} \\
24 \mathrm{HC}\end{array}$ & 27 (9) days & $\begin{array}{l}\text { mTBI } 36(12) \\
\text { HC } 37(10)\end{array}$ & $\begin{array}{l}\text { mTBI } 60 \% \\
\text { females } \\
\text { HC } 67 \% \\
\text { females }\end{array}$ & $\begin{array}{l}\text { DTI } \\
\text { Post-concussion } \\
\text { symptoms } \\
\text { (including fatigue } \\
\text { but not specifically } \\
\text { measured). }\end{array}$ & $\begin{array}{l}\text { mTBI reported more } \\
\text { post-concussion symptoms, did } \\
\text { not differ on cognitive tests, and } \\
\text { had a larger number of low DTI } \\
\text { measures (fractional anisotropy } \\
\text { values, } p=0.003 \text { ) compared } \\
\text { to controls. }\end{array}$ \\
\hline
\end{tabular}

Abbreviations: sd (standard deviation), mTBI (mild Traumatic Brain Injury), HC (healthy controls), OC (orthopedic controls), fMRI (functional Magnetic Resonance Imaging), rCBF SPECT (regional Cerebral Blood Flow Single-Photon emission computed tomography), ALS (arterial spin labeling), fNIRS (functional Near-Infrared Spectroscopy), DTI (Diffusion Tensor Imaging), GM (grey matter), WM (white matter), SDMT (Symbol Digit Modality Test), MFI (Multidimensional Fatigue Inventory), FSS (Fatigue Severity Scale), MFS (Mental Fatigue Scale), VAS (Visual Analogue Scale).

In summary, the brain imaging results are not consistent, although they show altered activity and connectivity in relation to fatigue after TBI. From the fMRI and fNIRS fatigue studies above, experimental block or event-related designs were used. Results need to be understood in relation to the study design used, as the results can vary depending on design. Discordance between study designs was reported for brain tumor patients [81]. 
No studies relating glucose metabolism (FDG-PET[(18)F]fluorodeoxyglucose-positron emission tomography) or magnetoencephalography $(M E G)$ to fatigue in TBI were found, but it would be of interest to more deeply investigate energy metabolism in relation to fatigue after TBI and also to use MEG, a promising method for identifying mTBI [82].

\section{Suggestions for Future Research}

The heterogeneity was high between studies, with insufficient evidence to determine any cognitive or neuroimaging test to predict fatigue after TBI. However, the studies imply connections between cognitive function and brain activation in relation to fatigue after TBI. This is worth exploring further with an intention of being open-minded and considering explanations at many different levels.

Assessment of fatigue must be improved. Here, different fatigue assessment scales were used in the reports. The construction of the scale may have an impact on the results, depending on sensitivity and specificity. It is also important to differentiate between normal and pathological fatigue, as biological and psychological factors may have a different explanation for mental fatigue.

Mental fatigue and its relation to depression needs to be acknowledged after TBI, as it will be important for treatment strategies.

With regard to mental fatigue affecting everyday life, executive and cognitive function have not been explored adequately and it is important to reach a clearer understanding of these aspects in relation to fatigue.

On a group level, slower processing speed is encountered primarily in relation to mental fatigue. More demanding tests may be more sensitive in detecting fatigue, but there is no clear connection to fatigue. Endurance in everyday life is critical for those suffering from mental fatigue, and repetition of tests may be more valid for measuring fatigue.

Brain imaging methods show promising results, establishing an association between fatigue and brain activation, primarily in the frontal cortex, thalamus, caudate nucleus, and connectivity between these areas. Cognitive and physical functions can be related to focal brain injuries. When no single area is clearly affected and cognitive tests are performed within the spectrum of normality and yet debilitating mental fatigue persists, we need to investigate further. We need to better understand the impact of the neurochemical cascade that can induce inflammation, biochemical imbalance, oxidative stress, and an extended reduction of glucose metabolism in relation to mental fatigue [36]. Brain imaging is a growing research field along with other imaging methods such as PET and MEG, which also warrant investigation.

Mental fatigue is not a new phenomenon, and it is essential to acknowledge the debilitating fatigue that patients describe despite the limited availability of diagnostic criteria and the lack of a clear definition of mental fatigue after TBI or mTBI and its origins.

Funding: This research received no external funding.

Institutional Review Board Statement: Not applicable.

Informed Consent Statement: Not applicable.

Data Availability Statement: Not applicable.

Conflicts of Interest: The author declares no conflict of interest.

\section{References}

1. Belmont, A.; Agar, N.; Hugeron, C.; Gallais, B.; Azouvi, P. Fatigue and traumatic brain injury. Ann. Readapt. Med. Phys. 2006, 49, 283-288. [CrossRef]

2. Cantor, J.B.; Ashman, T.; Gordon, W.; Ginsberg, A.; Engmann, C.; Egan, M.; Spielman, L.; Dijkers, M.; Flanagan, S. Fatigue after Traumatic Brain Injury and Its Impact on Participation and Quality of Life. J. Head Trauma Rehabil. 2008, 23, 41-51. [CrossRef] [PubMed]

3. Olver, J.; Ponsford, J.L.; Curran, C.A. Outcome following traumatic brain injury: A comparison between 2 and 5 years after injury. Brain Inj. 1996, 10, 841-848. [CrossRef] [PubMed] 
4. Cassidy, J.D.; Carroll, L.J.; Peloso, P.M.; Borg, J.; Von Holst, H.; Holm, L.; Kraus, J.; Coronado, V.G. Incidence, risk factors and prevention of mild traumatic brain injury: Results of the who collaborating centre task force on mild traumatic brain injury. $J$. Rehabil. Med. 2004, 36, 28-60. [CrossRef] [PubMed]

5. Hawthorne, G.; Gruen, R.L.; Kaye, A.H. Traumatic brain injury and long-term quality of life: Findings from an Australian study. J. Neurotrauma 2009, 26, 1623-1633. [CrossRef] [PubMed]

6. Ahman, S.; Saveman, B.-I.; Styrke, J.; Björnstig, U.; Stålnacke, B.-M. Long-term follow-up of patients with mild traumatic brain injury: A mixed-method study. J. Rehabil. Med. 2013, 45, 758-764. [CrossRef]

7. Stéfan, A.; Mathé, J.-F. What are the disruptive symptoms of behavioral disorders after traumatic brain injury? A systematic review leading to recommendations for good practices. Ann. Phys. Rehabil. Med. 2016, 59, 5-17. [CrossRef] [PubMed]

8. Reitan, R.M.; Wolfson, D. The two faces of mild head injury. Arch. Clin. Neuropsychol. 1999, 14, 191-202. [CrossRef]

9. Cassidy, J.D.; Boyle, E.; Carroll, L.J. Population-Based, Inception Cohort Study of the Incidence, Course, and Prognosis of Mild Traumatic Brain Injury after Motor Vehicle Collisions. Arch. Phys. Med. Rehabil. 2014, 95, S278-S285. [CrossRef]

10. De Koning, M.E.; Scheenen, M.E.; Van Der Horn, H.J.; Hageman, G.; Roks, G.; Yilmaz, T.; Spikman, J.M.; Van Der Naalt, J. Outpatient follow-up after mild traumatic brain injury: Results of the UPFRONT-study. Brain Inj. 2017, 31, 1102-1108. [CrossRef]

11. McInnes, K.; Friesen, C.L.; MacKenzie, D.E.; Westwood, D.A.; Boe, S.G. Mild Traumatic Brain Injury (mTBI) and chronic cognitive impairment: A scoping review. PLoS ONE 2017, 12, e0174847. [CrossRef]

12. Brett, B.L.; Wu, Y.-C.; Mustafi, S.M.; Saykin, A.J.; Koch, K.M.; Nencka, A.S.; Giza, C.C.; Goldman, J.; Guskiewicz, K.M.; Mihalik, J.P.; et al. The Association Between Persistent White-Matter Abnormalities and Repeat Injury after Sport-Related Concussion. Front. Neurol. 2020, 10. [CrossRef]

13. van der Horn, H.J.; Out, M.L.; de Koning, M.E.; Mayer, A.R.; Spikman, J.M.; Sommer, I.E.; van der Naalt, J. An integrated perspective linking physiological and psychological consequences of mild traumatic brain injury. J. Neurol. 2019, 267, 2497-2506. [CrossRef]

14. Mollayeva, T.; Kendzerska, T.; Mollayeva, S.; Shapiro, C.M.; Colantonio, A.; Cassidy, J.D. A systematic review of fatigue in patients with traumatic brain injury: The course, predictors and consequences. Neurosci. Biobehav. Rev. 2014, 47, 684-716. [CrossRef]

15. Lannsjö, M.; Geijerstam, J.-L.A.; Johansson, U.; Bring, J.; Borg, J. Prevalence and structure of symptoms at 3 months after mild traumatic brain injury in a national cohort. Brain Inj. 2009, 23, 213-219. [CrossRef]

16. Cantor, J.B.; Gordon, W.; Gumber, S. What is post TBI fatigue? Neurorehabilitation 2013, 32, 875-883. [CrossRef] [PubMed]

17. Johansson, B.; Rönnbäck, L. Long-Lasting Mental Fatigue after Traumatic Brain Injury-A Major Problem Most Often Neglected Diagnostic Criteria, Assessment, Relation to Emotional and Cognitive Problems, Cellular Background, and Aspects on Treatment. Trauma. Brain Inj. 2014. [CrossRef]

18. Stulemeijer, M.; van der Werf, S.; Bleijenberg, G.; Biert, J.; Brauer, J.; Vos, P.E. Recovery from mild traumatic brain injury: A focus on fatigue. J. Neurol. 2006, 253, 1041-1047. [CrossRef] [PubMed]

19. Andelic, N.; CENTER-TBI Participants Investigators; Røe, C.; Brunborg, C.; Zeldovich, M.; Løvstad, M.; Løke, D.; Borgen, I.M.; Voormolen, D.C.; Howe, E.I.; et al. Frequency of fatigue and its changes in the first 6 months after traumatic brain injury: Results from the CENTER-TBI study. J. Neurol. 2021, 268, 61-73. [CrossRef]

20. Andersson, E.H.; Björklund, R.; Emanuelson, I.; Stålhammar, D. Epidemiology of traumatic brain injury: A population based study in western Sweden. Acta Neurol. Scand. 2003, 107, 256-259. [CrossRef]

21. O'Connor, C.; Colantonio, A.; Polatajko, H. Long Term Symptoms and Limitations of Activity of People with Traumatic Brain Injury: A Ten-Year Follow-up. Psychol. Rep. 2005, 97, 169-179. [CrossRef]

22. Bushnik, T.; Englander, J.; Wright, J. Patterns of Fatigue and Its Correlates over the First 2 Years after Traumatic Brain Injury. J. Head Trauma Rehabil. 2008, 23, 25-32. [CrossRef]

23. Chaudhuri, A.; Behan, P.O. Fatigue in neurological disorders. Lancet 2004, 363, 978-988. [CrossRef]

24. Skau, S.; Bunketorp-Käll, L.; Kuhn, H.G.; Johansson, B. Mental Fatigue and Functional Near-Infrared Spectroscopy (fNIRS)—Based Assessment of Cognitive Performance After Mild Traumatic Brain Injury. Front. Hum. Neurosci. 2019, 13. [CrossRef]

25. Berginström, N.; Nordström, P.; Ekman, U.; Eriksson, J.; Andersson, M.; Nyberg, L.; Nordström, A. Using Functional Magnetic Resonance Imaging to Detect Chronic Fatigue in Patients with Previous Traumatic Brain Injury: Changes Linked to Altered Striato-Thalamic-Cortical Functioning. J. Head Trauma Rehabil. 2018, 33, 266-274. [CrossRef] [PubMed]

26. Möller, M.C.; Nordin, L.E.; Bartfai, A.; Julin, P.; Li, T.-Q. Fatigue and Cognitive Fatigability in Mild Traumatic Brain Injury are Correlated with Altered Neural Activity during Vigilance Test Performance. Front. Neurol. 2017, 8, 496. [CrossRef] [PubMed]

27. Kohl, A.D.; Wylie, G.R.; Genova, H.M.; Hillary, F.G.; DeLuca, J. The neural correlates of cognitive fatigue in traumatic brain injury using functional MRI. Brain Inj. 2009, 23, 420-432. [CrossRef]

28. Wylie, G.R.; Dobryakova, E.; DeLuca, J.; Chiaravalloti, N.; Essad, K.; Genova, H. Cognitive fatigue in individuals with traumatic brain injury is associated with caudate activation. Sci. Rep. 2017, 7, 1-12. [CrossRef]

29. Nordin, L.E.; Möller, M.C.; Julin, P.; Bartfai, A.; Hashim, F.; Li, T.-Q. Post mTBI fatigue is associated with abnormal brain functional connectivity. Sci. Rep. 2016, 6, 21183. [CrossRef] [PubMed]

30. Haber, S.N.; Calzavara, R. The cortico-basal ganglia integrative network: The role of the thalamus. Brain Res. Bull. 2009, 78, 69-74. [CrossRef] [PubMed]

31. Rabinowitz, A.R.; Levin, H.S. Cognitive Sequelae of Traumatic Brain Injury. Psychiatr. Clin. N. Am. 2014, 37, 1-11. [CrossRef] 
32. Sundstrom, A.; Marklund, P.; Nilsson, L.-G.; Cruts, M.; Adolfsson, R.; Van Broeckhoven, C.; Nyberg, L. APOE influences on neuropsychological function after mild head injury: Within-person comparisons. Neurology 2004, 62, 1963-1966. [CrossRef] [PubMed]

33. Ponsford, J.; McLaren, A.; Schönberger, M.; Burke, R.; Rudzki, D.; Olver, J.; Ponsford, M. The Association between Apolipoprotein E and Traumatic Brain Injury Severity and Functional Outcome in a Rehabilitation Sample. J. Neurotrauma 2011, 28, 1683-1692. [CrossRef] [PubMed]

34. Johansson, B.; Berglund, P.; Rönnbäck, L. Mental fatigue and impaired information processing after mild and moderate traumatic brain injury. Brain Inj. 2009, 23, 1027-1040. [CrossRef]

35. Dobryakova, E.; Genova, H.M.; DeLuca, J.; Wylie, G.R. The Dopamine Imbalance Hypothesis of Fatigue in Multiple Sclerosis and Other Neurological Disorders. Front. Neurol. 2015, 6, 52. [CrossRef]

36. Giza, C.C.; Hovda, D.A. The neurometabolic cascade of conucssion. J. Athl. Train. 2001, 36, 228-235. [PubMed]

37. Rönnbäck, L.; Hansson, E. On the potential role of glutamate transport in mental fatigue. J. Neuroinflamm. 2004, 1, 22. [CrossRef]

38. Lacourt, T.E.; Vichaya, E.G.; Chiu, G.S.; Dantzer, R.; Heijnen, C.J. The High Costs of Low-Grade Inflammation: Persistent Fatigue as a Consequence of Reduced Cellular-Energy Availability and Non-adaptive Energy Expenditure. Front. Behav. Neurosci. 2018, 12, 78. [CrossRef] [PubMed]

39. Komura, A.; Kawasaki, T.; Yamada, Y.; Uzuyama, S.; Asano, Y.; Shinoda, J. Cerebral Glucose Metabolism in Patients with Chronic Mental and Cognitive Sequelae after a Single Blunt Mild Traumatic Brain Injury without Visible Brain Lesions. J. Neurotrauma 2019, 36, 641-649. [CrossRef] [PubMed]

40. Sundstrom, A.; Nilsson, L.; Cruts, M.; Adolfsson, R.; Van Broeckhoven, C.; Nyberg, L. Fatigue before and after mild traumatic brain injury: Pre-post-injury comparisons in relation toApolipoproteinE. Brain Inj. 2007, 21, 1049-1054. [CrossRef]

41. Ashman, T.A.; Spielman, L.A.; Hibbard, M.R.; Silver, J.M.; Chandna, T.; Gordon, W.A. Psychiatric challenges in the first 6 years after traumatic brain injury: Cross-sequential analyses of axis I disorders11No commercial party having a direct financial interest in the results of the research supporting this article has or will confer a benefit upon the authors(s) or upon any organization with which the author(s) is/are associated. Arch. Phys. Med. Rehabil. 2004, 85, 36-42. [CrossRef]

42. Lundin, A. Mild Traumatic Brain Injury: Clinical Course and Prognostic Factors for Postconcussional Disorder; Stockholm University Stockholm: Stockholm, Sweden, 2007.

43. Stulemeijer, M.; Vos, P.E.; Bleijenberg, G.; Van Der Werf, S.P. Cognitive complaints after mild traumatic brain injury: Things are not always what they seem. J. Psychosom. Res. 2007, 63, 637-645. [CrossRef]

44. Schiehser, D.M.; Delis, D.C.; Filoteo, J.V.; Delano-Wood, L.; Han, S.D.; Jak, A.J.; Drake, A.I.; Bondi, M.W. Are self-reported symptoms of executive dysfunction associated with objective executive function performance following mild to moderate traumatic brain injury? J. Clin. Exp. Neuropsychol. 2011, 33, 704-714. [CrossRef]

45. Bushnik, T.; Caplan, B.; Bogner, J.; Brenneret, L.; Ponsford, J.; Schönberger, M.; Rajaratnam, S.M.W. A Model of Fatigue Following Traumatic Brain Injury. J. Head Trauma Rehabil. 2015, 30, 277-282.

46. Juengst, S.; Skidmore, E.; Arenth, P.M.; Niyonkuru, C.; Raina, K.D. Unique Contribution of Fatigue to Disability in CommunityDwelling Adults with Traumatic Brain Injury. Arch. Phys. Med. Rehabil. 2013, 94, 74-79. [CrossRef] [PubMed]

47. Beaulieu-Bonneau, S.; Ouellet, M.-C. Fatigue in the first year after traumatic brain injury: Course, relationship with injury severity, and correlates. Neuropsychol. Rehabil. 2017, 27, 983-1001. [CrossRef] [PubMed]

48. Johansson, B.; Rönnbäck, L. Evaluation of the Mental Fatigue Scale and its relation to Cognitive and Emotional Functioning after Traumatic Brain Injury or Stroke. Int. J. Phys. Med. Rehabil. 2014, 2, 182.

49. Fisher, L.B.; Pedrelli, P.; Iverson, G.L.; Bergquist, T.F.; Bombardier, C.H.; Hammond, F.M.; Hart, T.; Ketchum, J.M.; Giacino, J.; Zafonte, R. Prevalence of suicidal behaviour following traumatic brain injury: Longitudinal follow-up data from the NIDRR Traumatic Brain Injury Model Systems. Brain Inj. 2016, 30, 1311-1318. [CrossRef] [PubMed]

50. Nguyen, S.; McKay, A.; Wong, D.; Rajaratnam, S.M.; Spitz, G.; Williams, G.; Mansfield, D.; Ponsford, J.L. Cognitive Behavior Therapy to Treat Sleep Disturbance and Fatigue After Traumatic Brain Injury: A Pilot Randomized Controlled Trial. Arch. Phys. Med. Rehabil. 2017, 98, 1508-1517.e2. [CrossRef] [PubMed]

51. Johansson, B.; Bjuhr, H.; Rönnbäck, L. Mindfulness-based stress reduction (MBSR) improves long-term mental fatigue after stroke or traumatic brain injury. Brain Inj. 2012, 26, 1621-1628. [CrossRef] [PubMed]

52. Lezak, M.D.; Howieson, D.B.; Loring, D.W. Neuropsychological Assessment, 4th ed.; Oxford University Press: New York, NY, USA, 2004.

53. Bigler, E.D. Neuropsychology and clinical neuroscience of persitent post-concussive syndrome. J. Int. Neuropshychol. Soc. 2008, 14, 1-22. [CrossRef]

54. Cicerone, K.; Levin, H.; Malec, J.; Stuss, D.; Whyte, J. Cognitive Rehabilitation Interventions for Executive Function: Moving from Bench to Bedside in Patients with Traumatic Brain Injury. J. Cogn. Neurosci. 2006, 18, 1212-1222. [CrossRef]

55. Chan, R.C.K.; Shum, D.H.K.; Toulopoulou, T.; Chen, E.Y.H. Assessment of executive functions: Review of instruments and identification of critical issues. Arch. Clin. Neuropsychol. 2008, 23, 201-216. [CrossRef] [PubMed]

56. Spitz, G.; Ponsford, J.L.; Rudzki, D.; Maller, J.J. Association between cognitive performance and functional outcome following traumatic brain injury: A longitudinal multilevel examination. Neuropsychology 2012, 26, 604-612. [CrossRef] [PubMed]

57. Wilson, L.; Horton, L.; Kunzmann, K.; Sahakian, B.J.; Newcombe, V.F.; Stamatakis, E.A.; von Steinbuechel, N.; Cunitz, K.; Covic, A.; Maas, A.; et al. Understanding the relationship between cognitive performance and function in daily life after traumatic brain injury. J. Neurol. Neurosurg. Psychiatry 2021, 92, 407-417. [CrossRef] 
58. Sbordone, R.J. Neuropsychological Tests are Poor at Assessing the Frontal Lobes, Executive Functions, and Neurobehavioral Symptoms of Traumatically Brain-Injured Patients. Psychol. Inj. Law 2010, 3, 25-35. [CrossRef]

59. Ashman, T.A.; Cantor, J.B.; Gordon, W.A.; Spielman, L.; Egan, M.; Ginsberg, A.; Engmann, C.; Dijkers, M.; Flanagan, S. Objective Measurement of Fatigue Following Traumatic Brain Injury. J. Head Trauma Rehabil. 2008, 23, 33-40. [CrossRef] [PubMed]

60. Azouvi, P.; Couillet, J.; Leclercq, M.; Martin, Y.; Asloun, S.; Rousseaux, M. Divided attention and mental effort after severe traumatic brain injury. Neuropsychology 2004, 42, 1260-1268. [CrossRef]

61. Belmont, A.; Agar, N.; Azouvi, P. Subjective fatigue, mental effort, and attention dificits after severe traumatic brain injury. Neurorehabilit. Neural Repair 2009, 23, 939-944. [CrossRef]

62. Ziino, C.; Ponsford, J. Selective attention deficits and subjective fatigue following traumatic brain injury. Neuropsychology 2006, 20, 383-390. [CrossRef]

63. Ziino, C.; Ponsford, J. Vigilance and fatigue following traumatic brain injury. J. Int. Neuropsychol. Soc. 2006, 12, 100-110. [CrossRef]

64. Möller, M.C.; De Boussard, C.N.; Oldenburg, C.; Bartfai, A. An investigation of attention, executive, and psychomotor aspects of cognitive fatigability. J. Clin. Exp. Neuropsychol. 2014, 36, 716-729. [CrossRef]

65. Borgaro, S.R.; Baker, J.; Wethe, J.V.; Prigatano, G.P.; Kwasnica, C. Subjective Reports of Fatigue During Early Recovery From Traumatic Brain Injury. J. Head Trauma Rehabil. 2005, 20, 416-425. [CrossRef] [PubMed]

66. Anderson, J.F.I.; Cockle, E. Investigating the Effect of Fatigue and Psychological Distress on Information Processing Speed in the Postacute Period After Mild Traumatic Brain Injury in Premorbidly Healthy Adults. Arch. Clin. Neuropsychol. 2021, 4. [CrossRef]

67. Möller, M.C.; Johansson, J.; Matuseviciene, G.; Pansell, T.; DeBoussard, C.N. An observational study of trait and state fatigue, and their relation to cognitive fatigability and saccade performance. Concussion 2019, 4, CNC62. [CrossRef] [PubMed]

68. Borgaro, S.R.; Gierok, S.; Caples, H.; Kwasnica, C. Fatigue after brain injury: Initial reliability study of the BNI fatigue scale. Brain Inj. 2004, 18, 685-690. [CrossRef]

69. Johansson, B.; Rönnbäck, L. Novel computer tests for identification of mental fatigue after traumatic brain injury. Neurorehabilitation 2015, 36, 195-202. [CrossRef]

70. Rau, T.F.; Patel, S.A.; Guzik, E.E.; Sorich, E.; Pearce, A.J. Efficacy of a repeat testing protocol for cognitive fatigue assessment: A preliminary study in postconcussive syndrome participants. Concussion 2017, 2, CNC44. [CrossRef]

71. Pearce, A.J.; Tommerdahl, M.; King, D.A. Neurophysiological abnormalities in individuals with persistent post-concussion symptoms. Neuroscience 2019, 408, 272-281. [CrossRef]

72. Liu, K.; Li, B.; Qian, S.; Jiang, Q.; Li, L.; Wang, W.; Zhang, G.; Sun, Y.; Sun, G. Mental fatigue after mild traumatic brain injury: A 3D-ASL perfusion study. Brain Imaging Behav. 2016, 10, 857-868. [CrossRef]

73. Ramage, A.E.; Tate, D.F.; New, A.B.; Lewis, J.D.; Robin, D.A. Effort and Fatigue-Related Functional Connectivity in Mild Traumatic Brain Injury. Front. Neurol. 2019, 9, 1165. [CrossRef] [PubMed]

74. Hattori, N.; Swan, M.; Stobbe, G.A.; Uomoto, J.M.; Minoshima, S.; Djang, D.; Krishnananthan, R.; Lewis, D.H. Differential SPECT Activation Patterns Associated with PASAT Performance May Indicate Frontocerebellar Functional Dissociation in Chronic Mild Traumatic Brain Injury. J. Nucl. Med. 2009, 50, 1054-1061. [CrossRef]

75. Schonberger, M.; Reutens, D.; Beare, R.; O'Sullivan, R.; Rajaratnam, S.M.; Ponsford, J. Brain lesion correlates of fatigue in individuals with traumatic brain injury. Neuropsychol. Rehabil. 2016, 27, 1056-1070. [CrossRef]

76. Berginström, N.; Nordström, P.; Nyberg, L.; Nordström, A. White matter hyperintensities increases with traumatic brain injury severity: Associations to neuropsychological performance and fatigue. Brain Inj. 2020, 34, 415-420. [CrossRef] [PubMed]

77. Wäljas, M.; Lange, R.T.; Hakulinen, U.; Huhtala, H.; Dastidar, P.; Hartikainen, K.; Öhman, J.; Iverson, G.L. Biopsychosocial Outcome after Uncomplicated Mild Traumatic Brain Injury. J. Neurotrauma 2014, 31, 108-124. [CrossRef]

78. Clark, A.L.; Delano-Wood, L.; Sorg, S.F.; Werhane, M.L.; Hanson, K.L.; Schiehser, D.M. Cognitive fatigue is associated with reduced anterior internal capsule integrity in veterans with history of mild to moderate traumatic brain injury. Brain Imaging Behav. 2017, 11, 1548-1554. [CrossRef]

79. Clark, A.L.; Sorg, S.F.; Holiday, K.; Bigler, E.D.; Bangen, K.J.; Evangelista, N.D.; Bondi, M.W.; Schiehser, D.M.; Delano-Wood, L. Fatigue Is Associated with Global and Regional Thalamic Morphometry in Veterans with a History of Mild Traumatic Brain Injury. J. Head Trauma Rehabil. 2018, 33, 382-392. [CrossRef] [PubMed]

80. Pardini, M.; Krueger, F.; Raymont, V.; Grafman, J.H. Ventromedial prefrontal cortex modulates fatigue after penetrating traumatic brain injury. Neurology 2010, 74, 749-754. [CrossRef] [PubMed]

81. Tie, Y.; Suarez, R.O.; Whalen, S.; Radmanesh, A.; Norton, I.H.; Golby, A.J. Comparison of blocked and event-related fMRI designs for pre-surgical language mapping. NeuroImage 2009, 47, T107-T115. [CrossRef]

82. Antonakakis, M.; Dimitriadis, S.I.; Zervakis, M.; Papanicolaou, A.C.; Zouridakis, G. Aberrant Whole-Brain Transitions and Dynamics of Spontaneous Network Microstates in Mild Traumatic Brain Injury. Front. Comput. Neurosci. 2020, 13. [CrossRef] [PubMed] 